$$
\begin{aligned}
& \text { AUS DER ABTEILUNG FÜR } \\
& \text { PSYCHOSOMATISCHE MEDIZIN, } \\
& \text { PROF. DR. THOMAS LOEW } \\
& \text { DER FAKULTÄT FÜR MEDIZIN } \\
& \text { DER UNIVERSITÄT REGENSBURG }
\end{aligned}
$$

\title{
Psychotherapieausbildung in Europa
}

Inaugural-Dissertation zur Erlangung des Doktorgrades der Medizin

$$
\text { der }
$$

Fakultät für Medizin

der Universität Regensburg

$$
\text { vorgelegt von }
$$

Sebastian Kaufmann 



$$
\begin{aligned}
& \text { AUS DER ABTEILUNG FÜR } \\
& \text { PSYCHOSOMATISCHE MEDIZIN, } \\
& \text { PROF. DR. THOMAS LOEW } \\
& \text { DER FAKULTÄT FÜR MEDIZIN } \\
& \text { DER UNIVERSITÄT REGENSBURG }
\end{aligned}
$$

\section{Psychotherapieausbildung in Europa}

Inaugural-Dissertation zur Erlangung des Doktorgrades der Medizin

$$
\text { der }
$$

Fakultät für Medizin

der Universität Regensburg

vorgelegt von

Sebastian Kaufmann 
Dekan:

1. Berichterstatter:

2. Berichterstatter:

Tag der mündlichen Prüfung: 8. Dezember 2014
Prof. Dr. Dr. Torsten E. Reichert Prof. Dr. Thomas Loew Prof. Dr. Karin Tritt 
Inhalt

I. Zusammenfassung der Dissertation

1) Einleitung

2) Methodik

3) Ergebnisse und Diskussion

4) Eigene Ergebnisse im Vergleich zur aktuellen Literatur

5) Ausblick

6) Anhang

7) Quellen

II. Originalarbeit: Kaufmann, S., Tritt, K., Loew, Th. (2012) Psychotherapieausbildung in Europa, PDP 2012; 11: 207-219 


\section{Zusammenfassung der Dissertation}

\section{Einleitung}

In Deutschland ist die Ausbildung der nichtärztlichen Psychotherapeuten seit 1998 gesetzlich geregelt (Bundesministerium der Justiz, 2007). Bei den ärztlichen Psychotherapeuten gelten die jeweiligen landesspezifischen Weiterbildungsordnungen der Ärztekammern. Bisher existiert keine umfassende Untersuchung, die die diesbezügliche Situation in unseren europäischen Nachbarländern im Vergleich qualitativ und quantitativ gegenüberstellt. Ausgangspunkt ist, dass in Europa derzeit steigende Prävalenzraten psychischer Erkrankungen mit einhergehendem Bedarf an Psychotherapie beobachtet werden (Lademann, Mertesacker, \& Gebhardt, 2006) (Wittchen \& Jacobi, 2005). Es gibt Hinweise darauf, dass die Qualität der Ausbildung das Therapieergebnis maßgeblich beeinflussen kann (Fernandez-Liria, Rodriguez-Vega, Ortiz-Sanchez, Baldor Tubet, \& Gonzalez-Juarez, 2010). Auch in Deutschland wird aktuell über eine Reform der Psychotherapeutenausbildung heftig diskutiert, in der Schweiz ist eine solche vor kurzem beschlossen worden. Es scheint daher durchaus notwendig, eine aktuelle ausbildungsorientierte Übersicht über die Psychotherapie in Europa zu geben. Die Fragestellung beinhaltet auch eine standespolitische Komponente. Zumindest in Mitteleuropa gibt es immer wieder Diskussionen über die Abrechnungsmöglichkeiten psychotherapeutischer Leistungen durch die verschiedenen Berufsgruppen. Da auch die Ausbildungen teils sehr unterschiedlich sind, wird dies oftmals in die Argumentation miteinbezogen.

Deutschland nimmt mit dem Facharzt für Psychosomatische Medizin und Psychotherapie zudem eine Sonderrolle im europäischen Vergleich ein. Gleiches gilt auch für die Abrechnungsmöglichkeiten verschiedener Psychotherapiemodalitäten. In Deutschland findet sich diesbezüglich 
momentan eine hohe Vergütung pro Stunde Psychotherapie zu Lasten der Krankenkassen, aber auf der anderen Seite eine spezifische Beschränkung, die eine Abrechnung nur für bestimmte Therapieansätze ermöglicht. Um eine fruchtbare Diskussion über diese Themen zu führen, ist ein Vergleich mit den europäischen Nachbarn sinnvoll. Zudem gibt es bereits seit längerem Bestrebungen die Ausbildung von Psychotherapeuten in einen europäischen Standard (European Certificate of Psychotherapy) zu passen (European Association for Psychotherapy, 2006). Auch um über ein solches Vorhaben sinnvoll diskutieren zu können, ist eine solide Datengrundlage über die Ist-Situation in den einzelnen Ländern unabdingbar. Dies wird umso wichtiger, als heute die Frage nach der Anerkennung von Ausbildungs- oder Studienabschlüssen aus dem Ausland auch eine reale Notwendigkeit auf den europäischen Arbeitsmärkten darstellt.

Vorarbeiten wurden bereits 2001 mit dem SEPT (The Survey Of European Psychotherapy Training) von van Deurzen et al. geleistet (Deurzen, 2001). Hierbei ging es vor allem um die Umsetzung der ERCP (European Certificate of Psychotherapy) Kriterien in den jeweiligen Ländern zum damaligen Zeitpunkt. Damals wurde mit Fragebogen gearbeitet. Ebenfalls im Jahr 2001 wurden auch von Prof. Wolfgang Senf in seiner Funktion als Präsident der International Federation of Psychotherapy (IFP) Fragebögen mit dem Ziel, die Ausübung der Psychotherapie im internationalen Vergleich zu untersuchen, verschickt. Bei Präsentation der Ergebnisse lag die Rücklaufquote noch bei unter $10 \%$ (Senf, 2001). Zudem hatte van Deurzen bereits 2002 die Ausbildung in einigen Ländern mit den Kriterien des European Certificate of Psychotherapy der EAP (European Association of Psychotherapy) verglichen. 2002 legte Alfred Pritz das bisherige Standardwerk der Psychotherapie im internationalen Vergleich vor (Pritz, 2002). 2011 folgte eine weitreichende Darstellung von Situation und Ausbildung der Psychotherapie mehrerer europäischer Länder in der Zeitschrift European Psychotherapy, in der von jeweiligen nationalen 
Experten einzelne Artikel verfasst wurden (Sulz \& Hagspiegel (ed.), 2011). Vorliegende Arbeit möchte das Bild weiter ergänzen, erstmalig den finanziellen Aspekt hinzufügen, sowie die Unterschiede ärztlicher und nicht-ärztlicher Psychotherapeuten in Europa darstellen. Es wird in unserer strukturierten, interviewbasierten Befragung explizit nach einem Unterschied bezüglich der Ausbildung ärztlicher und nicht-ärztlicher Therapeuten gefahndet. Dies ist auf die bereits beschriebene deutsche Sonderstellung des Facharztes für Psychosomatik und Psychotherapie sowie die über 85-jährige Tradition ärztlicher Psychotherapie zurückzuführen.

Methodik

Zur Gewinnung der Daten wurden ein Interviewleitfaden und ein ergänzender Fragebogen in deutscher sowie englischer Sprache erstellt, welche die Eckpunkte einer psychotherapeutischen Ausbildung erfassen: u.a. Gesamtdauer, Selbsterfahrung, Supervision, Zugangsvoraussetzungen. Im Verlauf der Untersuchung und auch im Vergleich mit der aktuellen Literatur (Sulz \& Hagspiegel, 2011) hatte sich gezeigt, dass mit diesen Items die Ausbildung in jedem untersuchten Land beschrieben werden kann und auch bei anderen, vergleichbaren Arbeiten danach gefragt wurde. Weiterhin wurden die Gesamt- und Teilkosten der Ausbildung, sowie die Therapiekosten und ggf. deren Erstattung für den Patienten abgefragt. Aufgrund geringer Rücklaufquoten bei vorhergehenden Projekten wurde vor die Versendung des Fragebogens eine telefonische Kontaktaufnahme gesetzt. Bei der Recherche geeigneter Kontaktpersonen wurde auf das Kontaktverzeichnis für nationale Delegierte der European Association for Psychotherapy, auf die großen nationalen Fachverbände für Psychotherapie sowie auf Universitäten zurückgegriffen. Dies stellte in der Praxis eine große Herausforderung dar, da die entsprechenden Verzeichnisse oft nicht aktuell waren, Personen 
zwischenzeitlich nicht mehr die angegebenen Positionen innehatten oder die Kontaktdaten schlicht veraltet waren. Die wichtigste Ressource für Kontaktdaten stellte ohne Zweifel die Internetrecherche dar. Hierbei stellte jedoch die Vielfalt der europäischen Sprachen einen limitierenden Faktor dar. Zuweilen hat es sich als hilfreich erwiesen, einen praktizierenden Therapeuten im jeweiligen Land zu kontaktieren und direkt zu den entsprechenden Verbänden oder Regierungsstellen zu befragen. Sehr hilfreich waren auch die Einzelkapitel zu den Ländern in Pritz' European Psychotherapy (Pritz, 2002), da hier etliche nationale Verbände, teils auch mit Kontaktdaten aufgeführt sind. Insgesamt hat es sich auch bei unserer Recherche gezeigt, dass "blind“ versendete e-mails meist nicht beantwortet werden. Auch eine vorherige telefonische Kontaktaufnahme war keine Garantie für einen ausgefüllten Fragebogen. In der Praxis waren zuvor terminierte und telefonisch geführte Interviews am erfolgreichsten. Im direkten Gespräch konnten missverständliche Fragen erläutert und Länderspezifika dargelegt werden. Dies war bei Fragebögen, die per e-mail zurückgeschickt worden waren, nicht möglich, so dass dann oft Rückfragen gestellt wurden, die den Prozess der Informationsgewinnung zusätzlich verkomplizierten. Inhaltlich blieb der Fragebogen während der gesamten Untersuchung gleich, während im Text kleinere Änderungen erfolgten, beziehungsweise Erläuterungen hinzugefügt wurden.

In Ländern ohne nationale Regelung werden lediglich die Daten von einem oder mehreren der großen psychotherapeutischen Fachverbänden angegeben. Diese Einschränkung schien aufgrund der Vielzahl von Therapiemodalitäten mit entsprechenden Verbänden unumgänglich. Zur besseren Vergleichbarkeit wurde versucht, in solchen Fällen die Ausbildung der Ausrichtungen mit breiter internationaler Akzeptanz, Historie und wissenschaftlicher Begründung - Psychoanalyse und Verhaltenstherapie - darzustellen. Für Malta wurde die Ausbildung an einem Gestalt Institut beschrieben, da dieses zum Zeitpunkt der Datenerhebung die einzige Ausbildungsstätte des Landes war. 
Die Werte der Ausbildungskosten sind nicht absolut, sondern orientierend zu verstehen, da bei der Erhebung nicht jedes einzelne Ausbildungsinstitut eines Landes berücksichtigt werden konnte. Bei Angaben in anderen Währungen als Euro wurde ein Stichtag für den Kurswert festgesetzt, um Schwankungen durch wirtschaftliche Einflüsse gering zu halten. Jedoch konnte ein gewisser zeitlicher Spielraum hinsichtlich der Datenerhebung nicht vermieden werden.

\section{Ergebnisse und Diskussion}

Die Präsentation der Ergebnisse der Arbeit erfolgt nach Ländern gegliedert mit jeweils im Kontext präsentierten Ergebnissen. Im Laufe der Datenerhebung hatte sich gezeigt, dass unser Fragebogen Items abfragte, die in der deutschen Regelung zur Ausbildung von Psychotherapeuten sehr genau, teils auch gesetzlich geregelt sind, jedoch in anderen Ländern zum Zeitpunkt der Datenerhebung mangels verbindlicher Regelungen teilweise so nicht erhoben werden konnten. Dies hatte zur Folge, dass wie bereits erwähnt, Empfehlungen oder Mitgliedsvoraussetzungen von Fachverbänden recherchiert und angegeben wurden. Dies gilt unter anderem auch für die Mindestdauer der Ausbildung und die Anzahl der Supervisions- und Selbsterfahrungsstunden. In manchen Ländern werden Ausbildungsgänge angeboten, die in der Dauer mit Jahren angegeben werden können, in anderen Ländern gibt es nur Vorgaben für Inhalte, zum Beispiel Anzahl der Theoriestunden, die in Teilzeit oder Vollzeit absolviert werden können. Ein direkter Vergleich der Ausbildungsdauer war somit nur eingeschränkt möglich. Dies gilt umso mehr, da es oft nicht eine allgemeine Ausbildung zum Psychotherapeuten gibt, sondern die abgefragten Items für verschiedene Therapiemodalitäten sehr unterschiedlich sind und zudem nicht alle Modalitäten für alle Länder erhoben werden konnten. Aus diesen Gründen war auch ein direkter Vergleich oder ein Ranking mittels eines 
Scores nicht sinnvoll. Man hätte auf diese Weise zum Beispiel den Organisationsgrad bezüglich der Psychotherapieausbildung in den verschiedenen Ländern vergleichen können. Nichtsdestotrotz war die Erhebung der Kenndaten der Ausbildungsgänge notwendig. Hierbei konnte auch die bereits 2001 von van Deurzen aufgestellte These einer Tendenz hin zu einer europäischen Einheit bezüglich der Ziele und Inhalte der Psychotherapeutenausbildung gestützt werden (Deurzen, 2001). In allen untersuchten Ländern beinhaltet die Ausbildung Supervision, Selbsterfahrung und theoretische Kenntnisse. Hinsichtlich der Gesamtdauer der Ausbildung sind die Unterschiede absolut betrachtet gering. Unterschiede ergeben sich, da die Ausbildungen teils in Teilzeit absolviert werden, teils als Studium in Vollzeit konzipiert sind. Der Median liegt hier bei 8 Jahren, wenn man geforderte Zugangsvoraussetzungen mit einbezieht, und bei 4 Jahren, betrachtet man ausschließlich die psychotherapeutische Ausbildung. Einschränkend muss jedoch hinzugefügt werden, dass es auch in Ländern mit gesetzlicher Regelung zur Psychotherapie Ausnahmen gibt. Beispielsweise sei hier die deutsche Regelung der Ausübung der Heilkunde ohne ärztliche Approbation im Rahmen des Heilpraktikergesetzes erwähnt, bei der auch ohne Studienabschluss eine therapeutische Ausbildung absolviert und entsprechende Leistungen - zum Teil sogar mit privaten Krankenkassen abrechenbar - angeboten werden können. Die Dauer der Ausbildung (ohne Zugangsvoraussetzung) liegt bei 11 von 18 Ländern bei 3-4 Jahren. Nur 3 Länder (Österreich, Slowenien, Tschechische Republik) gehen darüber hinaus, wobei Slowenien und Österreich jeweils mit dem Propädeutikum doch wieder eine Art Zugangsvoraussetzung einbeziehen und die Ausbildung in der Tschechischen Republik in Teilzeit erfolgt. 4 Länder (Lettland, Frankreich, Spanien, Griechenland) bleiben unterhalb von 3 Jahren. Bei Lettland ist die Dauer von 2,5 Jahren Vollzeitstudium auf Kunsttherapie begrenzt, in der Gruppenpsychotherapie sind es 3 Jahre. In Griechenland hat man die Wahl zwischen 2 Jahren Vollzeit- oder 4-5 Jahren Teilzeitausbildung. 
Sicherlich ist einer der eindrücklichsten Unterschiede zwischen den einzelnen Ländern die Zuordnung der Psychotherapie als Beruf oder Fachgebiet. Einige Länder, darunter auch Deutschland, verorten die Psychotherapie als Teilgebiet der Medizin oder Psychologie. Andere Länder, zum Beispiel Österreich, gewähren der Psychotherapie den Status einer eigenständigen Profession. Diese Unterschiede sind sicher im kulturhistorischen Kontext zu verstehen. Die sich ergebenden Konsequenzen sind jedoch enorm. So limitieren die Länder der ersten Gruppe den Zugang zur Psychotherapieausbildung auf Studienabsolventen der Medizin beziehungsweise der Psychologie und unter speziellen Voraussetzungen auf Pädagogen bei der Kinder- und Jugendlichenpsychotherapie. Die zweite Gruppe setzt meist nur einen höheren Schulabschluss voraus. Es ist durchaus vorstellbar, dass eine solche Vorgehensweise Unterschiede in der Heterogenität der Therapeutenpopulation zur Folge hat.

Eine eigene psychotherapeutische Fachausbildung für Ärzte gibt es in den meisten Ländern nicht. Ausnahmen stellen hier Deutschland und Lettland mit dem Facharzt für Psychosomatische Medizin und Psychotherapie dar. In allen anderen untersuchten Ländern durchlaufen Mediziner (mit Ausnahme der Psychiater innerhalb ihrer Facharztausbildung) die gleiche Ausbildung wie zum Beispiel auch Psychologen oder Pädagogen. Jedoch werden psychotherapeutische Leistungen in aller Regel auch von Psychiatern angeboten. Der psychotherapeutische Anteil dieser Facharztausbildungen in den einzelnen Ländern ist in dieser Arbeit nicht untersucht worden.

Wie in der Einleitung erwähnt, hat die vorliegende Arbeit auch die finanziellen Aspekte aufgegriffen; Kosten für die Ausbildung von Psychotherapeuten sowie die Kosten für therapeutische Leistungen wurden ebenfalls abgefragt. Dies stellt in der Reihe der bisherigen, vergleichbaren Publikationen eine Neuerung dar. Insgesamt war auch bei 
den Kosten eine hohe Bandbreite innerhalb Europas zu eruieren. In manchen Ländern ist eine komplette Ausbildung an öffentlichen Hochschulen möglich. Dort können allgemeine Studiengebühren anfallen. In der Mehrzahl der untersuchten Länder findet die Ausbildung jedoch an privaten Instituten statt. Die Kosten betragen meist mehrere 10.000 Euro. Ein West-Ost Gefälle bezüglich des Preisniveaus konnte prinzipiell nicht beobachtet werden. Setzt man die Kosten zum durchschnittlichen Jahreseinkommen ins Verhältnis, findet die teuerste Ausbildung in Litauen statt, gefolgt von Slowenien und Norwegen.

Von besonderer sozialpolitischer Bedeutung sind die Kosten für Psychotherapie, die dem einzelnen Patienten im jeweiligen Land entstehen, beziehungsweise die Frage nach der Erstattung durch das Gesundheitssystem. Hier zeigte sich eine erhebliche Variabilität von um 25 Euro pro Sitzung in den osteuropäischen Ländern bis 65 Euro in den westeuropäischen. Ebenfalls große Unterschiede ergaben sich bei der Frage nach der Kostendeckung für psychotherapeutische Leistungen. Diese müssen in der Praxis weitgehend vom Patienten selbst bezahlt werden. Alternativ können Patienten psychiatrische Leistungen innerhalb des öffentlichen Gesundheitssystems in Anspruch nehmen. Je nach nationaler Regelung werden diese Leistungen in der Regel von den gesetzlichen Krankenkassen bezahlt oder vom öffentlichen Gesundheitswesen erbracht. Anders verhält es sich, wenn Psychotherapie von niedergelassenen Psychotherapeuten angeboten wird. Eine anteilige/ vollständige Erstattung der Therapiekosten für die Patienten ist dann (unter Einschränkungen) nur in Großbritannien, Deutschland, Schweden, Finnland und Österreich möglich. Inwieweit dies insgesamt hinreichend ist, haben wir in dieser Arbeit nicht untersucht, wobei von beinahe allen Interviewpartnern lange Wartezeiten auf Psychotherapieplätze beschrieben wurden. Pritz bezeichnete 2011 in einem Editorial diese Situation sogar als Einschränkung des Rechts auf Zugang zu medizinischer und psychotherapeutischer Hilfe (Sulz \& Hagspiegel, 2011). 
Eigene Ergebnisse im Vergleich zur aktuellen Literatur

Die letzte große Publikation zu dem Thema Psychotherapieausbildung in Europa verfolgte hinsichtlich der Methodik einen alternativen Ansatz (Sulz \& Hagspiegel, 2011). In einer Sonderausgabe der European Psychotherapy wurde länderbezogen von nationalen Experten berichtet. Dies bringt den Vorteil, dass die Fragestellungen und Parameter automatisch im richtigen landesspezifischen Kontext verstanden und präsentiert werden, allerdings mit dem Nachteil der mangelnden Standardisierung. Demgegenüber tendiert ein Autor, der denselben Fragebogen für alle Länder erstellt und auswertet, dazu, die Daten mit dem ihm bekannten System zu vergleichen und entsprechend zu interpretieren. Weiterhin ist die Anzahl der benötigten Kontakte und Ressourcen ungleich höher.

In der Publikation in European Psychotherapy wurde die Situation der Psychotherapieausbildung in zehn Ländern genau beschrieben. Eines davon (Niederlande) wurde in unserer Arbeit nicht untersucht. Frankreich und Italien wurden untersucht und beschrieben, kommen jedoch in dem in Psychodynamische Psychotherapie erschienenen Artikel nicht vor. Sulz und Hagspiegel haben sich in ihrer Arbeit mit der Ausnahme von Polen auf Länder in Nord- und Westeuropa konzentriert. Für die in beiden Arbeiten beschriebenen Länder (Deutschland, Österreich, Spanien, Griechenland, Schweden, Vereinigtes Königreich) kann bei den Eckpunkten Übereinstimmung gesehen werden. Jedoch werden bisweilen aktuelle Entwicklungen oder politische Hintergründe in der Arbeit von Sulz und Hagspiegel genauer dargestellt oder unsere Darstellung ergänzt. So zum Beispiel für das Vereinigte Königreich, wo in unserer Arbeit die Regularien der UKCP angeführt wurden, bei Sulz und Hagspiegel jedoch der zweite große Verband PBC beschrieben wird (Hepburn, 2011). Am Ende der jeweiligen Länderartikel wird auch in der Publikation der 
European Psychotherapy eine kurze strukturierte Zusammenfassung gestellt, die die Eckpunkte der Ausbildung und Situation der Psychotherapie kurz zusammenfassen soll. Hier werden unter anderem die Dauer der Ausbildung, Zugangsvoraussetzung, Supervision und Selbsterfahrung aufgeführt, also die gleichen Items, die auch wir für unseren Fragebogen ausgewählt hatten. Im abschließenden Teil der Sonderausgabe beschreiben Sulz und Hagspiegel zusammenfassend ihre Ergebnisse. Auch hier weichen unsere Ergebnisse nicht ab. In beiden Arbeiten zeigt sich ein uneinheitliches Bild in Europa, was die Zugangsvoraussetzungen zur Ausbildung beziehungsweise Ausübung der Psychotherapie angeht. Trotz des momentan vorherrschenden bunten Bildes in Europa kommen Sulz und Hagspiegel, wir und bereits 2001 schon van Deurzen (Deurzen, 2001) zu dem Schluss, dass ein Trend zu einer staatlichen, straffen Regulierung in den Ländern stattfindet. Das letzte Land, das einen solchen Schritt unternommen hat, war 2011 die Schweiz.

Ein guter Überblick zur Thematik wurde 2009 auch von Strauß mit dem „Forschungsgutachten zur Psychotherapeutenausbildung“ vorgelegt (Strauß et al., 2009). Die Situation in Europa war in dieser Arbeit nur Teilaspekt. Methodisch wurde ebenfalls mit einem Fragebogen gearbeitet. Allerdings wurde dieser absichtlich nicht an Fachverbände, sondern an „Fachpersonen“ versendet. In der Arbeit werden die Daten von 23 Ländern dargestellt, allerdings nicht im länderspezifischen Kontext, sondern tabellarisch im Überblick. Der Tenor dieses Teilbereichs der Arbeit deckt sich mit Sulz und Hagspiegel und auch unseren Ergebnissen. Strauß beschreibt eine heterogene Situation in Europa. Nur wenige Länder (7 von 23) schützen den Titel des Psychotherapeuten, weniger als die Hälfte verfügen über eine gesetzliche Regelung. Allerdings ist jedoch in mehreren Ländern eine solche in Planung (Strauß et al., 2009). Strauß sieht ebenfalls eine Zweiteilung bezüglich der "Heimat" der Psychotherapie; entweder als Teilgebiet von Medizin und Psychologie 
oder als eigenständige Profession, was sich meist in den Zugangsvoraussetzungen widerspiegelt.

Ausblick

Die Auswertung unserer Daten hat gezeigt, dass Europa in Hinblick auf Psychotherapie in Praxis und Ausbildung in bestimmten Aspekten erhebliche nationale Unterschiede aufweist. Dies betrifft vor allem den Zugang zur Ausbildung, sowie den Zugang zur Therapie der Patienten. Auch die Regelung der Ausbildung wird in den einzelnen Ländern sehr unterschiedlich gehandhabt. Andererseits konnten bei den Eckdaten der Ausbildung viele Übereinstimmungen gesehen werden. Dies wird zum einen deutlich anhand ähnlicher Gesamtzeiten der Ausbildung, sowie dem beinahe ausnahmslosen Vorhandensein von Supervision und Selbsterfahrung. Auch planen mehrere Länder für die Zukunft eine gesetzliche Regelung. Angesichts steigender Prävalenzzahlen der psychischen Erkrankungen in Europa wird es zunehmend wichtig sein, für Patienten eine Sicherheit zu schaffen, die garantiert, dass Psychotherapeuten hinreichend gut ausgebildet sind. Eine solide Ausbildung der Psychotherapeuten ist zudem Basis für einen möglichen Erstattungsprozess der Therapiekosten durch öffentliche oder private Krankenkassen. Diese und andere Arbeiten haben gezeigt, dass trotz aller Heterogenität die Grundelemente der Ausbildung in den einzelnen Ländern recht ähnlich sind. Es wird die Aufgabe der Zukunft sein, zu prüfen, inwieweit eine diesbezügliche, einheitliche europäische Regelung möglich, wünschenswert und sinnvoll ist. Zudem sollten die Ausbildungsstrukturen auch dazu führen, dass in Zukunft genügend gleichermaßen qualifizierte Psychotherapeuten zur Verfügung stehen. 
Anhang

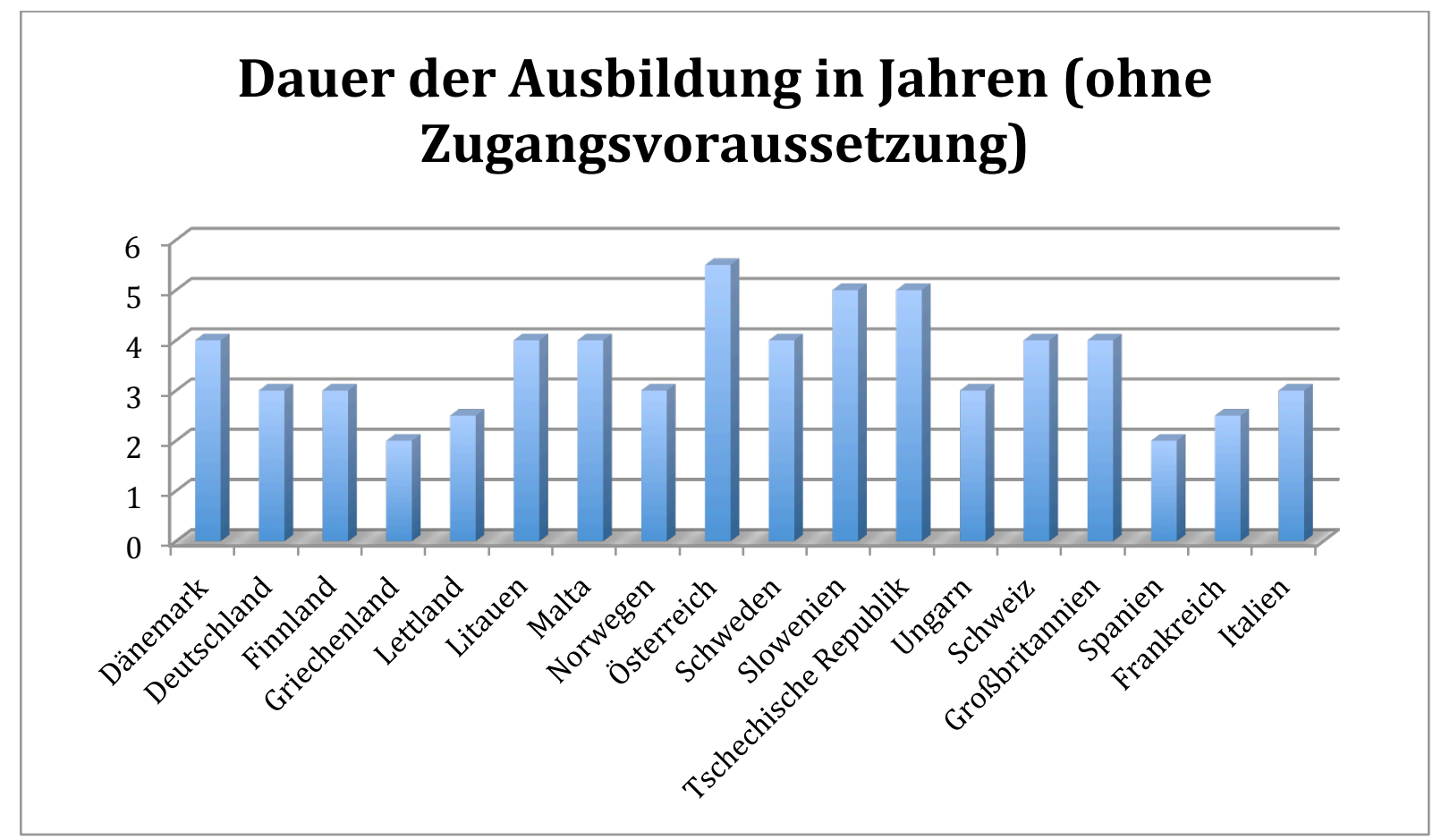

1) Österreich, Dänemark, Norwegen, Malta, Großbritannien in Teilzeit 


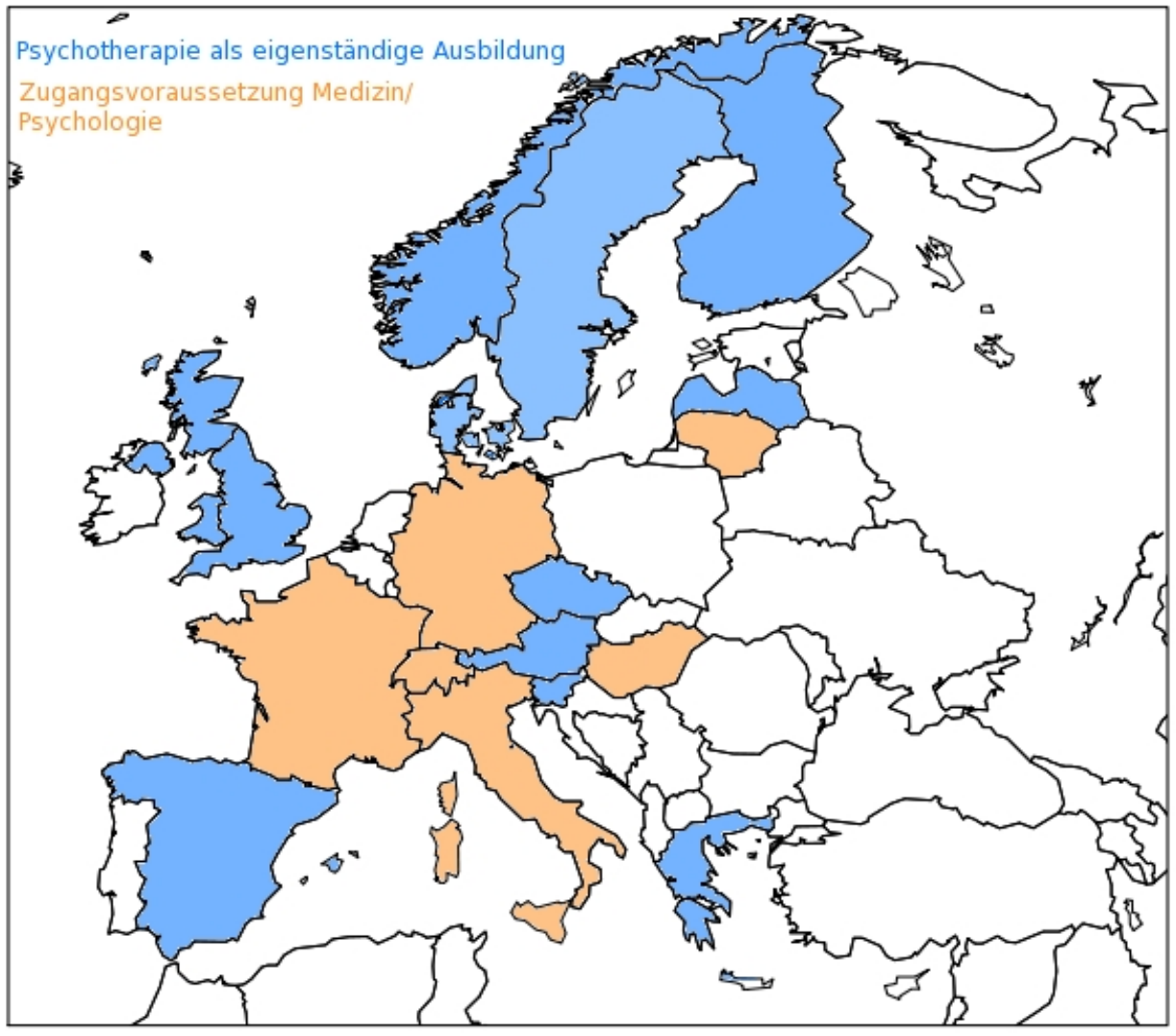

2) Zugangsvoraussetzung zur Psychotherapeutenausbildung 


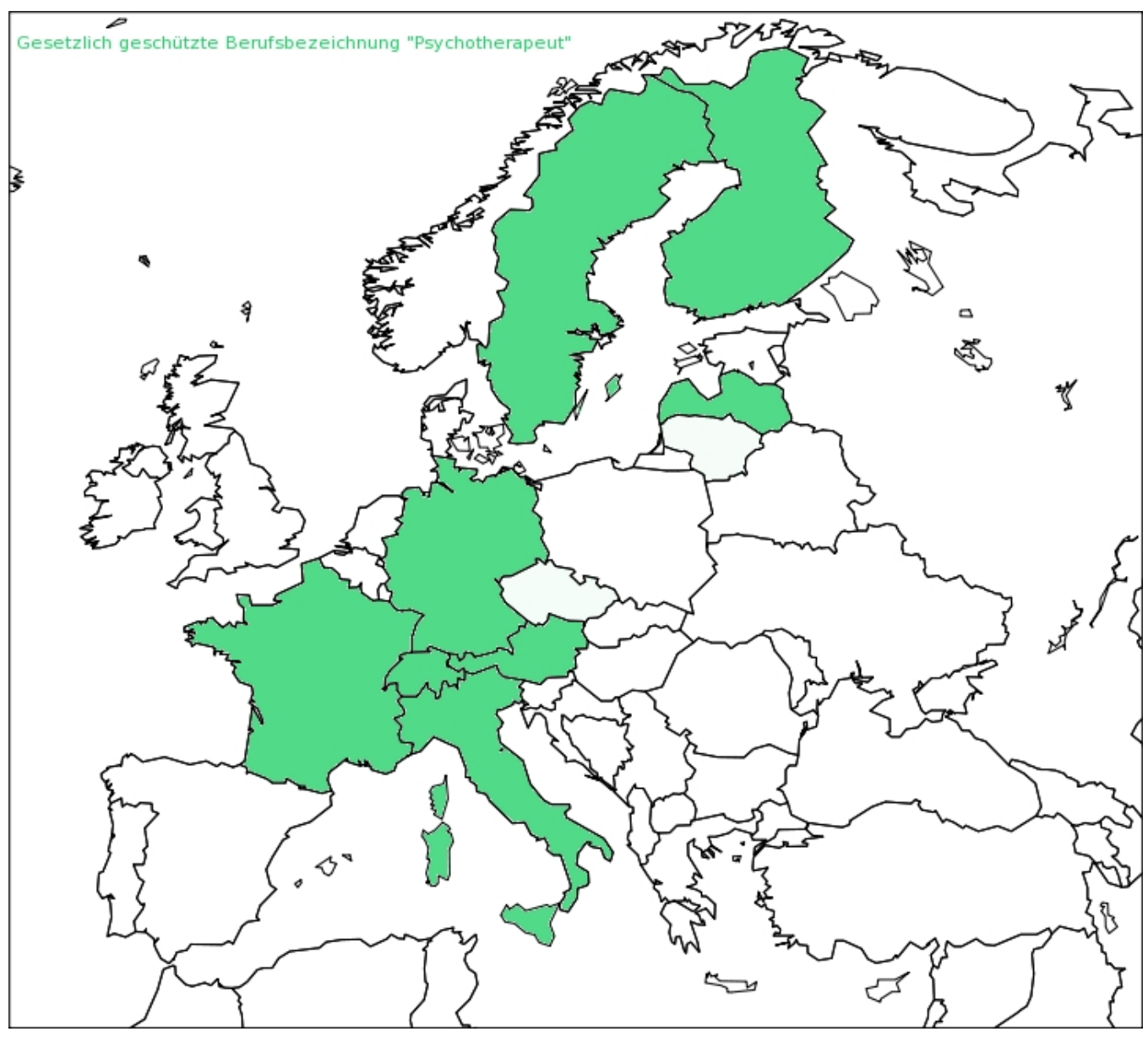

3) Gesetzlicher Schutz des Titels „Psychotherapeut“ 


\section{Quellen}

Bundesminsterium der Justiz. (2011). Gesetz über die Berufe des Psychologischen Psychotherapeuten und des Kinder- und Jugendlichenpsychotherapeuten (Psychotherapeutengesetz - PsychThG ). www.gesetze-im-internet.de, aufgerufen am 6.4.2014.

Bundesminsterium der Justiz. (2001). Gesetz über die berufsmäßige Ausübung der Heilkunde ohne Bestallung (Heilpraktikergesetz - HeilprG). www.gesetze-iminternet.de, aufgerufen am 6.4.2014.

Deurzen, E. V. (2001). Psychotherapy training in Europe: similarities and differences. Eur. J. of Psychotherapy, Counselling \& Health, 4(3), 357-371.

European Association for Psychotherapy (2006).The European Certificate for Psychotherapy.

Fernandez-Liria, A., Rodriguez-Vega, B., Ortiz-Sanchez, D., Baldor Tubet, I., \& Gonzalez-Juarez, C. (2010). Effectiveness of a structured training program in psychotherapeutic skills used in clinical interviews for psychiatry and clinical psychology residents. Psychotherapy research : journal of the Society for Psychotherapy Research, 20(1), 113-21.

Hepburn, J. M. (2011). Psychoanalytically oriented therapies in the UK - the current position. European Psychotherapy, 10, 134-159.

Lademann, J., Mertesacker, H., \& Gebhardt, B. (2006). Psychische Erkrankungen im Fokus der Gesundheitsreporte der Krankenkassen. Psychotherapeutenjournal,2, 123-129.

Pritz, A. (Ed. . (2002). Globalized Psychotherapy. (A. Pritz, Ed.). Vienna.

Senf, W. (2001). Dokumentation: internationale Situation der Psychotherapie.

Sulz, S. K. D. (ed), \& Hagspiegel, S. (ed. . (2011). Psychotherapy in Europe. European Psychotherapy, 10.

Strauß, B., Barnow, S., Brähler, E., Fegert, J., Fliegel, S., Freyberger, H. J., Goldbeck, L., et al. (2009). Forschungsgutachten zur Ausbildung von Psychologischen PsychotherapeutInnen und Kinder- und JugendlichenpsychotherapeutInnen.

Wittchen, H.U. \& Jacobi, F. (2005). Size and burden of mental disorders in Europe - a critical review and appraisal of 27 studies. European Neuropsychopharmacology, $15,357-37$ 
Die Originalarbeit wurde in „Psychodynamische Psychotherapie“, 2012: No. 4, S. 207-219 veröffentlicht und ist unter folgendem link abrufbar:

http://www.schattauer.de/de/magazine/uebersicht/zeitschriften-az/pdp/inhalt/archiv/issue/1608/manuscript/19060/show.html 